\title{
Protein crystallography analysis and ab initio structure determination with the new series of diffractometers from Rigaku Oxford Diffraction, the XtaLAB Synergy.
}

Pierre Le Magueres ${ }^{1}$, Angela R. Criswell ${ }^{1}$, Joseph D. Ferrara ${ }^{1}$.

Mathias Meyer ${ }^{2}$, Adrian Jones ${ }^{2}$, Przemyslaw Stec ${ }^{2}$ and Damian Kucharczyk².

${ }^{1}$ Rigaku Americas Corporation, 9009 New Tails Drive, The Woodland, Texas USA 77381-5209.

${ }^{2}$ Rigaku Oxford Diffraction, ul. Kazimierza Witalisa Szarskiego 3, 54-609 Wroclaw, Poland.

In the course of the past two years, activities at Rigaku Oxford Diffraction have given birth to a new series of single crystal X-ray diffractometers, the XtaLAB Synergy. As its name indicates, the XtaLAB Synergy combines the best of Rigaku and Oxford Diffraction in terms of hardware components, software programs and years of experience and savoir-faire in X-Ray crystallography on small molecule and protein compounds in the home lab.

Combining high X-Ray flux, multiple radiation wavelengths, very high data collection speed and proven easy-of-use of the control program CrysAlis ${ }^{\text {Pro }}$, the XtaLAB Synergy has been designed both for small molecule and protein work and features:

- A standardized and compact design that accommodates a rotating anode, a single wavelength or dual wavelength microfocus sealed tube X-Ray source without any change in its design.

- For the sealed tube version, a NEW PhotonJet-S series of microfocus sources available in Cu, Mo and Ag wavelengths that, combined to new optics, provide a significant increase of X-ray photons at the sample over previous generations of sealed tube microfocus sources.

- A completely redesigned kappa goniometer that allows for very fast data collection speed $(10 \%$ second), symmetrical $2 \theta$ positioning and omega collection scans in either direction.

- New hybrid photon counting detector from ROD, the HyPix-6000HE, featuring $100 \mu \mathrm{m}$ pixel size and a high frame rate of $100 \mathrm{~Hz}$ for very fast data collection.

- For protein work, the ability to decrease the beam divergence via a computer-controlled slit, allowing for reflection resolution along longer unit cell axes.

In this work, we focus on the usage of the XtaLAB Synergy for protein work and show how reflections along the long unit cell axis of thaumatin $(\sim 150 \AA)$ and catalase $(\sim 230 \AA)$ can be resolved, leading to structure solution for thaumatin by sulfur-SAD phasing and for catalase by molecular replacement at short detector distances. 